\title{
Investigation of pulsed spin-polarized electron beams at the S-DALINAC
}

\author{
Martin Espig* \\ Institut für Kernphysik, Technische Universität Darmstadt \\ E-mail: mespig@ikp.tu-darmstadt.de

\section{Joachim Enders} \\ Institut für Kernphysik, Technische Universität Darmstadt \\ E-mail: enderseikp.tu-darmstadt.de

\section{Yuliya Fritzsche} \\ Institut für Kernphysik, Technische Universität Darmstadt \\ E-mail: yuliya@ikp.tu-darmstadt.de
}

\section{Markus Wagner}

Institut für Kernphysik, Technische Universität Darmstadt

E-mail: mwagneraikp.tu-darmstadt.de

\begin{abstract}
A source of polarized electrons has been implemented at the superconducting Darmstadt electron linac S-DALINAC. It uses photo emission from strained-layer superlattice-GaAs (SSL) and bulk-GaAs photocathodes, driven by either $3 \mathrm{GHz}$ modulated diode lasers at $780 \mathrm{~nm}$ (high polarization) and $405 \mathrm{~nm}$ (unpolarized, high quantum efficiency) or a short-pulse Ti:Sapphire laser system at $780 \mathrm{~nm}$. We present results from measurements with varying laser pulse lengths obtained using a pulse stretcher system and a single-mode fiber, yielding electron bunch lengths between $4 \mathrm{ps}$ and $25 \mathrm{ps}$. The electron bunch length was determined using a chopper rf cavity and a slit system. The electron polarization along the temporal profile of the bunch was studied for SSL and bulk cathodes. The extracted data at low quantum efficiency require an extension of the model description for photo-emission from semiconductor cathodes.
\end{abstract}

Supported by DFG within CRC634 and by the state of Hesse through the LOEWE center HIC for FAIR.

XVth International Workshop on Polarized Sources, Targets, and Polarimetry,

September 9-13, 2013

Charlottesville, Virginia, USA

\footnotetext{
* Speaker.
} 


\section{Generation of pulsed electron beams}

The source of polarized electrons at the superconducting Darmstadt electron linac S-DALINAC [1] relies on photo emission from strained-layer superlattice-GaAs and bulk-GaAs photocathodes. To prepare a low-energy electron beam for radio-frequency accelerators, RF structure must be imposed on the beam. One way to do this relies on extracting a DC beam from the photocathode, and then passing this beam through a chopper cavity, providing a $\mathrm{TM}_{110}$ mode [2]. In this manner, $11 \%$ of the electron beam passes through a slit downstream. The resulting transmitted electron beam has rf-structure with bunchlength of 36,6 ps. A prebuncher system then compresses the bunch through a $\mathrm{TM}_{010}$ mode to a length of less than 5 ps. Hence, by extracting a continuous DC beam from the polarized source, $89 \%$ of the electrons are lost at the chopper-slit. This inefficient use of the polarized beam results in a short operating lifetime. It is better to create RF structure directly at the photocathode by using a pulsed laser system.

The lifetime and the quantum efficiency (QE) of the photocathode decrease over time due to ion back bombardment and residual gases. The QE can be described by

$$
Q E(t)=Q E(t=0) \exp \left(-\left(\frac{1}{\tau_{V}}+\frac{1}{\tau_{S}}\right) t\right)=\frac{I_{e l} h c}{e P_{L} \lambda} \exp \left(-\left(\frac{1}{\tau_{V}}+\frac{I_{e l}}{n_{e}}\right) t\right)
$$

where $I_{e l}$ represents beam current, and $P_{L}$ the average laser power at the laser wavelength $\lambda$. For the S-DALINAC polarized source the vacuum lifetime was $\tau_{V}=(1164 \pm 165) \mathrm{h}$ after extracting $(9,6 \pm 0,7) \mathrm{C}$, for initial $Q E$ of $2 \mu \mathrm{A} / \mathrm{mW}$ at $780 \mathrm{~nm}$ [3]. The decrease in QE must be compensated by an increase in laser power $P_{L}$ during an experiment. For the parameters at the S-DALINAC, the maximum power of $1 \mathrm{~W}$ should allow $60 \mu \mathrm{A}$ for one week, provided all emitted electrons are accelerated. There are two laser systems that can be used to create RF structure directly on the beam, to facilitate the efficient use of the extracted beam and thereby prolong the operating lifetime of the photogun.

\subsection{Diode laser}

Gain-switched diode lasers are modulated with a harmonic $3 \mathrm{GHz}$ signal, so that the repetition rate of the laser and the accelerator are the same. The behavior of different diode lasers were studied by variation of the direct current and the modulation power $[4,5]$. Diode lasers in an external cavity and a wavelength of $780 \mathrm{~nm}$ are used for high polarization experiments. Electron bunch lengths with a minimum pulse length of about $50 \mathrm{ps}$ and a peak to background ratio of about 10 were reached with this system. For unpolarized high current experiments blue diode lasers with $405 \mathrm{~nm}$ were used with approximately 22 ps long pulses [6].

\subsection{Ti:sapphire laser system}

A Ti:sapphire laser system operates at a wavelength of $780 \mathrm{~nm}$ for the generation of highly polarized electrons. This system has a repetition rate of $75 \mathrm{MHz}$, the 40th subharmonic of the S-DALINAC's fundamental frequency and generates pulse lengths from (200-400) fs by passive 
mode locking. These laser pulses are transported to the polarized source through a $50 \mathrm{~m}$ long optical fiber. The laser pulses are stretched due to the positive dispersion of the fiber. This effect is partially compensated by an optical pulse compressor, which generates adjustable negative dispersion. In this manner, laser pulse lengths between 4 ps and 25 ps were obtained [7]. For unpolarized high current experiments the Ti:sapphire laser can be frequency doubled to $390 \mathrm{~nm}$.

\section{Investigation of pulsed electron beams}

\subsection{Experimental setup}

The same $3 \mathrm{GHz}$ chopper cavity that prepares a DC beam for further acceleration is used for the measurement of the shape and the length of the bunches. As the longitudinal component of the pulse is transferred in the transverse space, the time measurement is realized through sampling the bunch as a function of phase shifts. The portion of the bunch that passes through a $100 \mu \mathrm{m}$ wide slit is delivered to a Faraday cup (figure 1). The resolution of this setup is $(7 \pm 3,5)$ ps, which is primary limited by the geometry [6]. For a phase resolved measurement of the polarization of the electron bunch, a Mott-polarimeter is used instead of the Faraday cup.

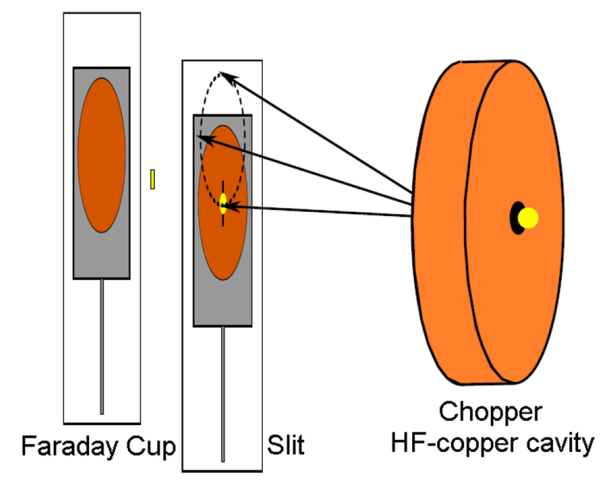

Figure 1: Principle of the electron bunch length measurement: the bunch (yellow) passes the chopper cavity and will be deflected with respect to its phase. The electrons that pass the slit are measured with a Faraday cup [6].

\subsection{Bunch length and polarization measurements of SSL and bulk GaAs photocathodes}

Electron bunches from strained-layer superlattice-GaAs (SSL) and bulk GaAs photocathodes were characterized using the Ti:sapphire laser with 28 ps pulses, with results shown in figure 2 . It is important to note that the studies were carried out with photocathodes that exhibits a low quantum efficiency. The full width at half maximum of both bunches was determined to about 28 ps. The bunch of the SSL cathode has a Gaussian shape. The first electrons that come out of the cathode are highly polarized. The Mott scattering asymmetry, and therefor the polarization, decreases fast along the temporal profile of the bunch. The dip in-polarization near the intensity maximum is, caused by a pile up effect in the Mott polarimeter data acquisition system. The bunch profile of the bulk cathode exhibits a long tail. Attributed to electrons originating from deep within the cathode, that require a long time to reach the surface. The Mott scattering asymmetry of the first electrons is 
high, too, but it decreases more slowly in time compared to the SSL cathode. To increase average polarization, the latest electrons of the bunch must be cut out.
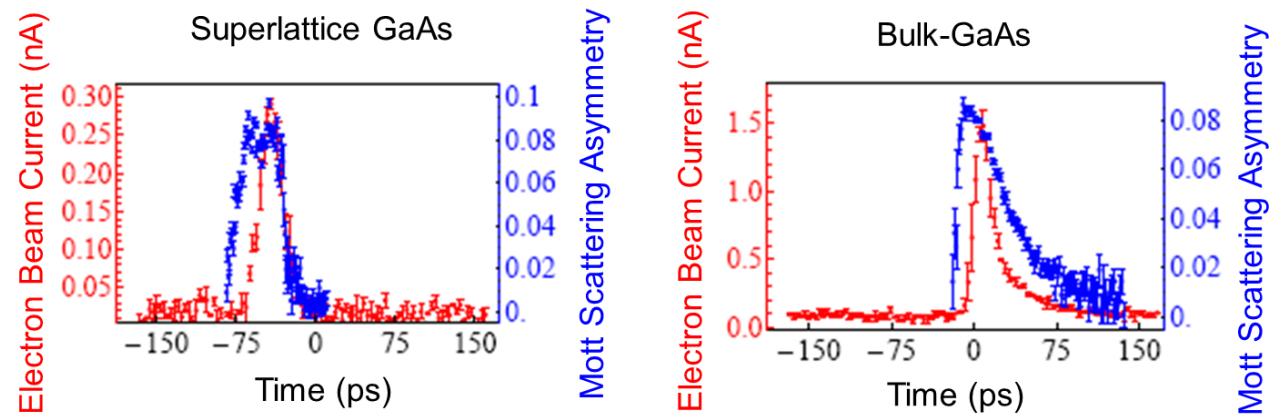

Figure 2: Comparison of the bunch shape and the polarization in time of a SSL and a bulk GaAs cathode. Both measurements were made with the Ti:sapphire laser system [6].

\section{Modeling of ultrashort electron bunches}

Usually, electron emission from GaAs photocathodes is described within the approach developed by Spicer [8]. Electrons in the conducting band diffuse to the cathode surface. During this phase they can recombine with holes in the valence band. Spicer described this with the rate equation

$$
\frac{\partial \Delta n(\vec{x}, t)}{\partial t}=g(\vec{x}, t)-\frac{\Delta n(\vec{x}, t)}{\tau}+D \nabla^{2} \Delta n(\vec{x}, t)
$$

with the electron density $\Delta n(\vec{x}, t)=\Delta n^{+}(\vec{x}, t)+\Delta n^{-}(\vec{x}, t)$ in the conduction band of the semiconductor, the generating laser pulse $g(\vec{x}, t)$, the average residence time $\tau$ in the conduction band and the diffusion constant $D$ of electrons in the GaAs crystal. This model was applied to the measured electron bunches, as shown in figure 3. The discrepancy between measured and predicted bunch shape indicates that the rate equation (3.1) is insufficient to describe the data. One explanation of this discrepancy is the poor QE of the cathodes. This results in a much higher electron concentration within the semiconductor for the same output current. In this case, recombination effects in the semiconductor may become important. This speculation is explored using an advanced model with additional recombination terms which consider optical recombination, Auger-processes and Shockley-Read-Hall-recombination [6]. Additionally, an attempt was made to more accurately describe the temporal polarization profile. Due to various depolarization mechanisms the polarization falls with time. To understand this effect, a spin-dependent particle model has been constructed that does not only simulates the pulse profile, but represents the polarization characteristics [6]:

$$
\begin{aligned}
& \frac{\partial \Delta n^{+/-}}{\partial t}=\overbrace{\gamma^{+/-} g(x, y, z, t)}^{\text {Laser }}-\overbrace{\left(A+B p_{0}\right) \Delta n^{+/-}}^{\text {SRH-Recombination }}-\overbrace{K\left(\Delta n^{+/-}-\Delta n^{-/+}\right)}^{\text {Spin-Flip }} \\
& -\overbrace{B \Delta n^{+/-}\left(\Delta n^{+/-}+\Delta n^{-/+}\right)}^{\text {opt. Recombination }}+\overbrace{D \nabla^{2} \Delta n^{+/-}}^{\text {Diffusion }}
\end{aligned}
$$

Here $\Delta n^{+/-}$are the particle spin densities. $\gamma^{+}=1-\gamma^{-}$describes the relative number of polarized electrons. For SSL cathodes $\gamma^{+}=1$, which represents a theoretical possible polarization of $100 \%$. 


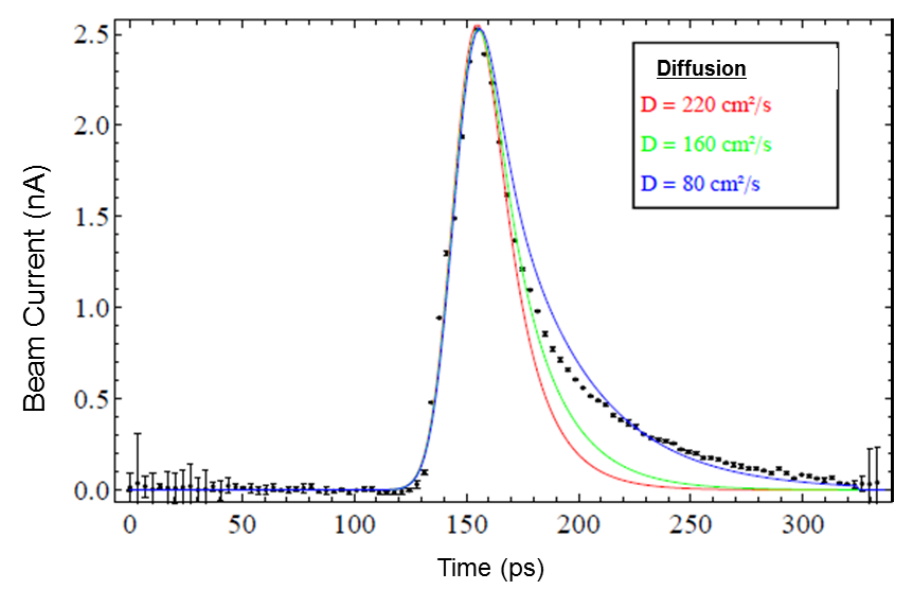

Figure 3: Electron bunch profile of a bulk GaAs-cathode with low quantum efficiency and three Spicer model fits with different diffusion constants [6].

For bulk cathodes $\gamma^{+}=0,75$, which represents a maximum polarization of $50 \%$. The ground state density of the holes in the valence band without laser irradiation is given by $p_{0}$. The diffusion constant $D$ is assumed to be $200 \mathrm{~cm}^{2} / \mathrm{s}$ [12].

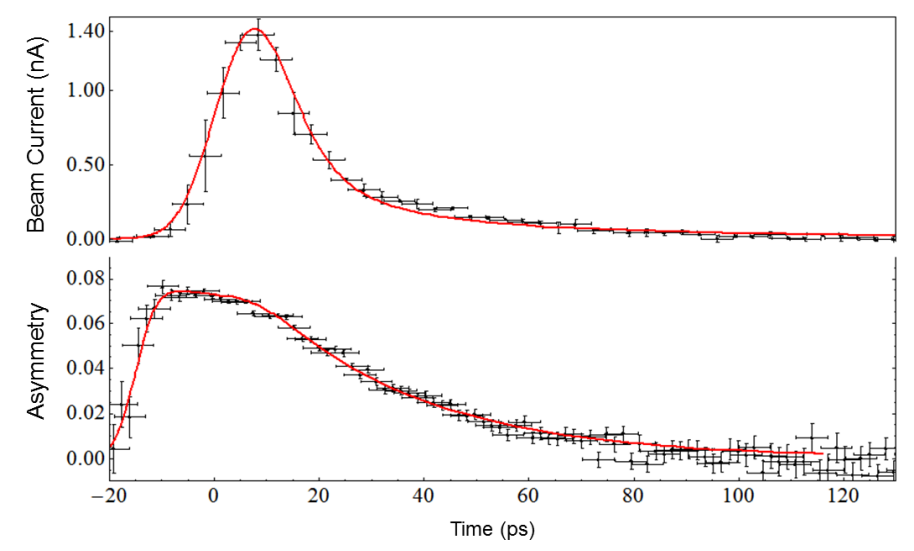

Figure 4: Electron bunch and polarization profile of a bulk GaAs-cathode with advanced model fits [6].

This expanded model is capable of describing the experimental data (figure 4) with the following parameters [6]:

- $1 / A=(30 \pm 10) \mathrm{ps}$

The SRH-constant $1 / A$ is small in comparison to the literature of 100 ps to some nanoseconds [9]. A possible explanation for this was presented in reference [10]: Due to a high electron current density, the parameter $1 / A$ becomes time-dependent. This may arise from low QE. The parameter also decreases in case of impurities on the cathode surface.

- $B=(1,0 \pm 2,7) \cdot 10^{-10} \mathrm{~cm}^{3} / \mathrm{s}$

The value of the recombination rate $B$ is in good agreement with the values reported in the 
literature [11]. The high level of uncertainty comes from the inaccurate determination of the particle density $n_{0}$.

- $C=(5,0 \pm 4,7) \cdot 10^{-28} \mathrm{~cm}^{6} / \mathrm{s}$

Comparing the Auger constant $C$ with the literature values [11] yields a difference about a factor of 5 . However, due to the large uncertainty, caused by the determination of the particle density $n_{0}$, the experimental result is not in conflict with the literature values.

- $1 / K=(61,6 \pm 2,0) \mathrm{ps}$

The lifetime of the spin-polarization $1 / K$ can be compared to the values of $(75 \pm 4)$ ps measured by reference [13]. This value is strongly correlated to the SRH-parameter.

Future measurements will be aimed at increasing the accuracy of the above listed parameters.

\section{References}

[1] Y. Fritzsche et al., Proc. Pol. Sources, Targets, and Polarimeters, St. Petersburg, 2011

[2] T. Bahlo, Bachelor Thesis, Technische Universität Darmstadt, 2010

[3] Y. Fritzsche, Dissertation D17, Technische Universität Darmstadt, 2011

[4] M. Espig, Master Thesis, Technische Universität Darmstadt, 2011

[5] A. Kaiser, Bachelor Thesis, Technische Universität Darmstadt, 2013

[6] M. Wagner, Dissertation D17, Technische Universität Darmstadt, 2013

[7] J. Lindemann, Master Thesis, Technische Universität Darmstadt, 2013

[8] W. E. Spicer, Phys. Rev. 112, 114, 1958

[9] C. J. Hwang, J. of Appl. Phys. 42, 4408, 1971

[10] E. F. Schubert, MSc Systems Engineering Optoelektronik II, FernUniversität Hagen, 2007

[11] Y. Arafat et al., Int. J. Optoelectron. 2, 5, 2012

[12] C. Gutsche et al., Nano Letters 12, 1453, 2012

[13] A. Oertel et al., Appl. Phys. Lett. 93, 132112, 2008 